\title{
Comparative study between Endovascular versus Open surgical management of Infra- renal Aortic Aneurysm
}

M.A.Mansour, E.A.Abd El-Mabood, H.S.Afifi and M.M.Mesalam

General Surgery, Dept., Faculty of Medicine, Benha Univ., Benha, Egypt

E-Mail:Moemen.mesalam@gmail.com

\begin{abstract}
Background; a dilation of the blood arteries is referred to as an aneurysm. The infra-renal section of the aorta, often known as the abdominal aortic aneurysm, has the greatest incidence of Arterial aneurysms in the extracranial system. The major cause of arterial aneurysm disease-related death and morbidity is abdominal aortic aneurysms. As a goal and aim, to compare the EVAR and OSR of an infrarenal aortic aneurysm on an elective basis with respect to technical concerns and postoperative results, 30 patients suffering from infra-renal aneurysms were studied from July 2020 to July 2021 at Nasser Institute for Research Hospital, Cairo, Egypt, and the vascular unit of the Department of surgery at Benha University Hospital, Egypt. As a result, 39 patients were originally screened, 4 did not satisfy eligibility requirements, and 2 declined to participate; 3 were lost to follow-up, and 30 were eventually enrolled in the research, Conclusion: For infrarenal AAA, therapeutic methods like as EVAR and OSR have shown to be effective. There are many advantages of elective vasectomy, including the ability to do it under regional or local anaesthetic, shorter recovery time, and less strain on hospital resources. In the EVAR group, general anaesthesia was used far less often. The EVAR group had a substantially shorter operational time than the OSR group. Acute myocardial infarction occurred at a lower rate in the EVAR group than in the OSR group after surgery. EVAR has a lower 30-day mortality rate than OSR, despite the fact that the difference is minor. In comparison to OSR, EVAR had fewer cases of systemic and local problems.
\end{abstract}

Keywords: Endovascular, Open surgical, Infra-renal, Aortic Aneurysm.

\section{Introduction}

If left untreated, an abdominal aortic aneurysm (AAA) may have life-threatening implications. Until the advent of endovascular repair in the 1990s, open surgical repair (OSR) was the only option. When techniques improved and newer technology were incorporated into the available kits, EVAR, a less invasive procedure with promising results, became the treatment of choice for AAA.

Despite this, despite advancements in the procedure, OSR remains popular, particularly in acute situations or where anatomical factors need it [3].

\section{Patients and methods}

\subsection{Study population.}

From July 2020 to July 2021, a prospective study was conducted on 30 patients with infra-renal aortic aneurysms in Nasser institute for research hospital in Cairo, Egypt and vascular unit, Department of surgery, Benha university hospital.

Patients suitable for endovascular therapy were chosen according to these criteria:

\subsection{Inclusion criteria}

- Age: $\leq 75$ years old.

- Sex: males and females.

- Aneurysm size $\geq 5.5 \mathrm{~cm}$ in males $\& \geq 5 \mathrm{~cm}$ in females in fusiform shape, Or increased rate of growth of the aneurysm by more than $10 \mathrm{~mm}$ in 12 months in both sexes.

- Any size in saccular shape.

- Symptomatic aneurysm of any size or shape.

- Surgically fit patients concerning the cardiac and general condition.

- Patients who match the instructions for use (IFU) criteria.

\subsection{Exclusion criteria}

- Age $\geq 75$.

- Aneurysm size $<5.5 \mathrm{~cm}$ in males and $<5 \mathrm{~cm}$ in females.

- Surgically unfit patients: Congestive heart failure, recent myocardial infarction, recent stroke, chronic renal insufficiency.

- Ruptured abdominal aortic aneurysm.

- Patients who don't match the IFU criteria.

\subsection{Methods}

Patients with infra-renal aortic aneurysm who match inclusion criteria will be divided randomly into two groups:

- >Group A: 15 patients in this group will undergo Endovascular abdominal repair of A.A.A. in patients who match IFU.

- >Group B: 15 patients in this group will undergo Open Surgical repair (OSR) of A.A.A.

All patients will be subjected to the following

- Examination will be done including weight, blood pressure, heart rate, blood glucose, abdominal examination and a complete systemic examination.

\author{
2.5. Investigations \\ Full laboratory investigations including \\ - Complete blood picture \\ - Coagulation profile \\ - Renal functions test \\ - Liver Functions test \\ Special investigations \\ - CT angiography will be done on the whole aorta \\ for diagnosis of A.A.A. \\ - Echocardiography
}




\section{Ethical considerations}

- The data will be confidential.

- A written consent will be obtained from all patients after explaining the procedure and its complications.

\section{Results.}

This study was conducted in the period from July 2020 to July 2021, thirty-nine patients were initially assessed, six were excluded (4 did not meet the eligibility criteria and 2 refused to participate), three were lost to follow-up, and 30 were ultimately included in the study.

The study included 30 patients who were 10 female $(33.3 \%)$ and 20 male $(66.7 \%)$. The mean age of patients in the entire cohort was $61.8 \pm 7.1$ years, ranging from 45 to 5673 years. There were no significant differences between the two groups regarding the age and gender of the patients. Regarding associated morbidities, 21 patients $(70 \%)$ complained of hypertension, 11 patients $(36.7 \%)$ were confirmed to suffer from ischemic heart disease, 9 patients $(30 \%)$ with type- 2 diabetes mellitus, and 17 patients $(56.7 \%)$ were current smokers as shown in Figure 65. There were no significant differences between the two groups regarding the prevalence of comorbidities or smoking. Table (1).

In EVAR group, two patients (13.3\%) were submitted to the procedure under general anesthesia, eight patients $(53.3 \%)$ under regional anesthesia, and five patients (33.3) under local anesthesia. While in OSR group, all patients (100\%) were operated on under general anesthesia. There was a significant difference between the two groups $(\mathrm{p}<0.0001)$ regarding the type of anesthesia. The mean operative time in EVAR group was $148 \pm 26$ minutes versus $185 \pm 23$ minutes in the OSR group. The operative time was significantly shorter in EVAR group ( $\mathrm{p}=0.0003$ ) compared

to OSR group. There was a significant difference between the two groups $(\mathrm{p}=0.0002)$ regarding intraoperative blood transfusion in favor of EVAR group. Table (2)
In EVAR group, 10 patients $(66.7 \%)$ were admitted to the intensive care unit (ICU) with a median duration of ICU stay of 1 day (ranging between 1-10 days). While, in OSR group, all the patients $(100 \%)$ required ICU admission with the median duration of stay of three days (ranging between 2-7 days. There was a significant difference between the two groups $(\mathrm{p}=0.042)$ regarding postoperative

ICU admission in favor of EVAR group. Also, there was a significant difference between the two groups $(\mathrm{p}<0.0001)$ regarding postoperative ICU stay in favor of EVAR group. There was a significant difference between the two groups $(p=0.0001)$ regarding postoperative ward stay in favor of EVAR group. Two patients $(13.3 \%)$ in EVAR group required secondary intervention to the primary procedure versus

five patients $(33.3 \%)$ in the OSR group. The results of the two groups were comparable regarding this outcome. Table (3).

One case $(6.7 \%)$ of mortality was recorded in EVAR group versus two cases $(13.3 \%)$ in OSR group. Similarly, during the ICU admission, one patient $(6.7 \%)$ in EVAR group required invasive ventilation versus two patients in OSR group. Also, one patient $(6.7 \%)$ in EVAR group was complicated with acute myocardial infarction versus two patients $(13.3 \%)$ in OSR group. In addition, one patient $(6.7 \%)$ in EVAR group was complicated with lower extremity ischemia versus two patients (13.3\%) in OSR group. Table (4).

Regarding EVAR group, only one patient $(6.7 \%)$ was complicated with endoleak. Similarly, one patient in OSR group was complicated with hemorrhage at the site of repair. The number of cases of surgical site infection in EVAR group was two patients (13.3\%) versus four patients $(26.7 \%)$ in OSR group. Also, two cases $(13.3 \%)$ of wound dehiscence were recorded, both in OSR group. Besides, one patient (6.7\%) in EVAR group was reported to attain graft thrombosis versus two patients $(13.3 \%)$ in OSR group. No reported cases of aortoenteric fistula. Table

Table (1): Demographic and baseline data

\begin{tabular}{lccc}
\hline Variable & $\begin{array}{c}\text { EVAR } \\
(\mathrm{n}=15)\end{array}$ & $\begin{array}{c}\text { OSR } \\
(\mathrm{n}=15)\end{array}$ & P value \\
\hline Age (year), mean + SD & $61.1 \pm 7.2$ & $62.6 \pm 7.1$ & 0.57 \\
Sex (male : female) & $10: 5$ & $10: 5$ & 1 \\
Comorbidities, n (\%) & & & \\
Hypertension & $10(66.7)$ & $5(33.3)$ & 1 \\
IHD & $6(40)$ & $3(20)$ & 1 \\
Diabetes mellitus & $6(40)$ & $9(60)$ & 1 \\
Smoking & $8(53.3)$ & & \\
EVAR, endovascular aneurysm repair; OSR, open surgery repair; SD, standard \\
deviation; IHD, ischemic heart disease.
\end{tabular}


Table (2) Operative details.

\begin{tabular}{llll}
\hline Variable & $\begin{array}{l}\text { EVAR } \\
(\mathrm{n}=15)\end{array}$ & $\begin{array}{l}\text { OSR } \\
(\mathrm{n}=15)\end{array}$ & P value \\
\hline Anesthesia,n $(\%)$ & & & $<0.0001$ \\
- $\quad$ General & $2(13.3)$ & $15(100)$ & \\
- $\quad$ Regional & $8(53.3)$ & $0(0)$ & \\
- $\quad$ Local & $5(33.3)$ & $0(0)$ & \\
Operative time (min.), mean \pm SD & $148 \pm 26$ & $185 \pm 23$ & 0.0003 \\
Blood transfusion, n (\%) & $0(0)$ & $10(66.7)$ & 0.0002 \\
EVAR, endovascular aneurysm repair; OSR, open surgery repair; SD, standard deviation.
\end{tabular}

Table (3) Postoperative course.

\begin{tabular}{lccc}
\hline Variable & $\begin{array}{c}\text { EVAR } \\
(\mathrm{n}=15)\end{array}$ & $\begin{array}{c}\text { OSR } \\
(\mathrm{n}=15)\end{array}$ & P value \\
\hline $\begin{array}{l}\text { Postoperative ICU } \\
\text { admission, n (\%) }\end{array}$ & $10(66.7)$ & $15(100)$ & 0.042 \\
$\begin{array}{l}\text { ICU stay, median } \\
\text { (range) }\end{array}$ & $1(1-10)$ & $3(2-7)$ & $<0.0001$ \\
$\begin{array}{l}\text { Ward stay, median } \\
\text { (range) }\end{array}$ & $1(1-7)$ & $5(3-14)$ & 0.0001 \\
$\begin{array}{l}\text { Secondary } \\
\text { intervention, n (\%) }\end{array}$ & $2(13.3)$ & $5(33.3)$ & 0.39 \\
$\begin{array}{l}\text { EVAR, endovascular aneurysm repair; OSR, open surgery repair; SD, standard deviation; } \\
\text { ICU, intensive care unit. }\end{array}$ &
\end{tabular}

Table (4) Postoperative systemic adverse events.

\begin{tabular}{|c|c|c|c|}
\hline Variable & $\begin{array}{l}\text { EVAR } \\
(n=15)\end{array}$ & $\begin{array}{c}\text { OSR } \\
(n=15)\end{array}$ & $P$ value \\
\hline Mortality (within 30 days), n (\%) & $1(6.7)$ & $2(13.3)$ & 1 \\
\hline Postoperative intubation > $48 \mathrm{hr} ., \mathrm{n}(\%)$ & $1(6.7)$ & $2(13.3)$ & 1 \\
\hline Acute myocardial infarction, n (\%) & $1(6.7)$ & $2(13.3)$ & 1 \\
\hline Acute renal failure, $n(\%)$ & $0(0)$ & $0(0)$ & 1 \\
\hline Respiratory failure, n (\%) & $0(0)$ & $0(0)$ & 1 \\
\hline Stroke, n (\%) & $0(0)$ & $0(0)$ & 1 \\
\hline Spinal cord ischemia, n (\%) & $0(0)$ & $0(0)$ & 1 \\
\hline Lower extremity ischemia, n (\%) & $1(6.7)$ & $2(13.3)$ & 1 \\
\hline Colonic ischemia, $\mathbf{n}(\%)$ & $0(0)$ & $0(0)$ & 1 \\
\hline
\end{tabular}

Table (5) Postoperative local complications.

\begin{tabular}{lccc}
\hline Variable & $\begin{array}{c}\text { EVAR } \\
(\mathrm{n}=15)\end{array}$ & $\begin{array}{c}\text { OSR } \\
(\mathrm{n}=15)\end{array}$ & P value \\
\hline Endoleak/Bleeding, n (\%) & $1(6.7)$ & $1(6.7)$ & 1 \\
Surgical site infection, n (\%) & $2(13.3)$ & $4(26.7)$ & 0.65 \\
Wound dehiscence, $\mathbf{n}(\%)$ & $0(0)$ & $2(13.3)$ & 0.48 \\
Graft thrombosis, $\mathbf{n}(\%)$ & $1(6.7)$ & $2(13.3)$ & 1 \\
Aortoenteric fistula, n (\%) & $0(0)$ & $0(0)$ & 1 \\
EVAR, endovascular aneurysm repair; OSR, open surgery repair. & \\
\hline
\end{tabular}

\section{Discussion}

Old age, male gender, smoking, high blood pressure, a family history of the illness, and coronary artery disease are all risk factors for AAA.

Smokers in the 65-74 year age range had the greatest incidence of AAA, and those over 75 had the second-highest incidence of the disease (two-thirds of cases were over 75 years old) [4]. Because of the linked health problems and the high rate of current smokers in the group, this is likely.

Smoking is the most significant risk factor for the development of AAA, with males accounting for the majority of those who smoke. Less AAA was found in nations where cigarettes were not as popular [8 \& 9]. There was a link between smoking and more ruptured AAAs, as well as a bigger diameter. 
AAA develops at an earlier age in smokers who are still smoking than in nonsmokers or ex-smokers. Additionally, the danger increases with continued smoking [11].

There is also a clear male preponderance, which is in accordance with the well-known fact that the incidence is greater among men, with women having a propensity to more severe effects. One explanation for the male preponderance is that men are more likely to suffer from cardiovascular problems [12].

Despite the fact that the function of high blood pressure is debatable, it is recognised as a significant risk factor [10]. Patients with hypertension are more likely to be evaluated and investigated on a frequent basis, which may explain the disagreement [10].

Many cardiovascular illnesses include diabetes as a risk factor, although AAA has not been identified as one of those diseases yet. There was no conclusive evidence that diabetes was linked to AAA development. Furthermore, a research [6] found a link between the two illnesses that was mostly caused by the antidiabetic medication metformin.

The EVAR group used relatively little general anaesthetic (13.3 percent ). A better result is known to be linked with the administration of local or regional anaesthetic. However, in order to validate this finding, additional randomised studies must be designed [13].

A prior research found that the EVAR group's operational time was substantially reduced compared to the OSR group's, and both procedures took less time than was reported in the same study [14]. According to the present research, the average operational time was 148 minutes for the EVAR group, compared to 215 minutes in the prior study. Operative times were similarly long in the OSR group (mean time of 185 minutes). In contrast to the earlier research, which found that it took 264 minutes [14]. In the present research, the patients were on an elective basis, operated on smaller aneurysms, and completed exclusively by senior surgeons. This may explain the lower operational time.

The EVAR group did not need blood transfusion, while two-thirds of the OSR group's patients did. There was much less blood loss in the EVAR group compared to the OSR group, which was previously reported by Menezes and coauthors [14].

There was a greater incidence of postoperative ICU hospitalisation in the OSR group (100 percent of the patients). Yet, the frequency is still high in the EVAR group in spite of this (66.7 percent ). There's a chance this is related to the big intervention, regardless of the strategy used. It was less painful and took less time to perform the EVAR technique in comparison to the other groups.

The overall length of hospitalisation (ICU+ward) was previously reported [14]. The length of hospital stay was shorter in the OSR group than in the EVAR group in this research, which contrasted with the findings from the present study. A three-day hospital stay was the norm in the present research for patients in the EVAR group, compared to a nine-day stay in the prior study. A 10-day hospital stay was the norm in the OSR group, compared to an average of 9 days in the prior study[14]. Contrary findings in the prior research may be attributable to a greater incidence of complications in the EVAR group, despite this not being statistically significant. This study's shorter stay is likely due to decreased rates of postoperative mechanical ventilation in the EVAR group.

Even though it was somewhat higher than in the prior research [15], the secondary intervention rate in both groups is consistent with a previous study's (13.3 percent and 33.3 percent, respectively, against 11 percent and 27 percent in the EVAR and OSR groups).

Acute myocardial infarction occurred at a lower rate in the EVAR group than in the OSR group after surgery, despite the difference being negligible. Also, the findings for the EVAR and OSR groups were much greater (6.7 percent and 13.3 percent, respectively) than in the prior research (1.3 percent and 5.4 percent) [16].

Additionally, the present research found much more cases of lower extremity ischemia $(10 \%$ of the total cohort) than a prior study (approximately 2\%), which was likely due to the earlier study using a multicenter design. In addition, the findings of the present research and the previous study [17] were similar between the two groups.

EVAR has a lower 30-day mortality rate than OSR, despite the fact that the difference is minor. These results are in line with a previous study by Malas and coworkers. Nevertheless, according to a prior research, the rate was significantly lower in the EVAR group (1.3 percent for EVAR vs 3.7 percent for OSR, P0.001) than in the other groups.

Endoleak following EVAR was less common in the present research (6.7 percent) than it had been in previous studies (12 percent) [19]. The most common reason for subsequent intervention is an endoleak [20]. Endoleaks following open surgery are uncommon, although one has been documented [21]. Only one patient in the OSR group, on the other hand, had complications due to bleeding. The same outcomes are due to the fact that both procedures were carried out by experienced surgeons.

More people than previously thought were affected by SSI. The present research found a $13.3 \%$ incidence of EVAR, compared to a prior study's 2.5 percent [22]. When looking at the OSR group, the new research found an SSI incidence of $26.7 \%$, up from the prior study's $3.8 \%$. These findings make sense if we consider that the majority of the patients had diabetes, which necessitated a lengthier surgical duration and an extended stay in the intensive care unit. They were treated carefully, using culture and susceptibility testing as well as antibiotics as necessary. However, bedside drainage was needed in just two of the OSR patients.

Patients with wound dehiscence who needed bedside drainage had their wounds treated conservatively until secondary intention healed them, 
then they were monitored for further abdominal wall repair.

6.7 percent of patients had graft occlusions after EVAR, and 13.3 percent had them after OSR, whereas the published rate was only 2-4 percent [23]. This may be because of the study's tiny sample size, which skews the findings.

Aortoenteric fistulas have been observed in earlier investigations at a rate of less than $1 \%$ [24]. Because of the limited sample size, this was not revealed in our research.

There are many advantages of elective vasectomy, including the ability to do it under regional or local anaesthetic, shorter recovery time, and less strain on hospital resources.

OSR had a reduced frequency of systemic and local complications, but there was no statistically significant difference between the two treatments when it came to these problems after surgery.

\section{Conclusion}

When it comes to treating infrarenal AAA, EVAR and OSR have been shown to be effective. There are many advantages of elective vasectomy, including the ability to do it under regional or local anaesthetic, shorter recovery time, and less strain on hospital resources. In the EVAR group, general anaesthesia was used far less often. The EVAR group had a substantially shorter operational time than the OSR group. Acute myocardial infarction occurred at a lower rate in the EVAR group than in the OSR group after surgery. EVAR has a lower 30-day mortality rate than OSR, despite the fact that the difference is minor. In comparison to OSR, EVAR had fewer cases of systemic and local problems.

\section{References}

[1] J.Powell, M.Sweeting, P.Ulug, J.Blankensteijn, F.Lederle, J.Becquemin . EVAR-1, DREAM, OVER and ACE Trialists. Meta-analysis of individual-patient data from EVAR-1, DREAM, OVER and ACE trials comparing outcomes of endovascular or open repair for abdominal aortic aneurysm over 5 years. $\mathrm{Br}$ J Surg.vol.104,pp.166-178,2017.

[2] A.England and Mc R.Williams Endovascular aortic aneurysm repair (EVAR). Ulster Med J.vol. 82, pp.3-10,2013.

[3] A.Katsargyris, C.Lenhardt, Marques de P.Marino, B.Botos and E.Verhoeven Reasons for and Outcomes of Open Abdominal Aortic Repair in the Endovascular Era.vol.73,pp.417422, 2021.

[4] D.Howard, A.Banerjee, F J.airhead, A.Handa, L.Silver and P.Rothwell Oxford Vascular Study. Age-specific incidence, risk factors and outcome of acute abdominal aortic aneurysms in a defined population. $\mathrm{Br} \mathrm{J}$ Surg.vol.102, pp.907-915,2015.
[5] M.Johansson, P.Zahl, V.Siersma, K.Jørgensen, B.Marklund and J.Brodersen Benefits and harms of screening men for abdominal aortic aneurysm in Sweden: a registry-based cohort study. Lancet.vol.391, pp. 2441-2447,2018.

[6] N.Itoga, K.Rothenberg, P.Suarez, T.Ho, M.Mell, B.Xu . Metformin prescription status and abdominal aortic aneurysm disease progression in the U.S. veteran population. J Vasc Surg.vol.69, pp.710-716,2019.

[7] D.Li, A.Busch, H.Jin, E.Chernogubova, J.Pelisek, J.Karlsson . H19 induces abdominal aortic aneurysm development and progression. Circulation.vol.138,pp.1551-1568,2018.

[8] F.Gianfagna, G.Veronesi, M.Tozzi, A.Tarallo, R.Borchini, M.Ferrario . Prevalence of abdominal aortic aneurysms in the general population and in subgroups at high cardiovascular risk in Italy: results of the RoCAV population based study. Eur. J. Vasc. Endovasc. Surg.vol. 55,pp.633-639,2018.

[9] J.Laroche, F.Becker, J.Baud, G.Miserey, A.Jaussent, M.Picot . Dépistage échographique de l'anévrisme de l'aorte abdominale - les enseignements de Vésale [Ultrasound screening of abdominal aortic aneurysm: Lessons from Vesale . J Mal Vasc.vol. 40, pp. 340-349,2015.

[10] E.Altobelli, L.Rapacchietta, V. Profeta and R.Fagnano Risk Factors for Abdominal Aortic Aneurysm in Population-Based Studies: A Systematic Review and Meta-Analysis. Int $\mathbf{J}$ Environ Res Public Health.vol. 15,pp.11122805, 2018.

[11] K.Kent, R.Zwolak, N.Egorova, T.Riles, Manganaro : Analysis of risk factors for abdominal aortic aneurysm in a cohort of more than 3 million individuals. Journal of vascular surgery.vol.52, pp.539-548,2010.

[12] S.Thompson, H.Ashton, L.Gao, M.Buxton and R.Scott Multicentre Aneurysm Screening Study (MASS) Group. Final follow-up of the Multicentre Aneurysm Screening Study (MASS) randomized trial of abdominal aortic aneurysm screening. Br J Surg.vol. 99, pp.164956, 2012.

[13] R.Armstrong, Y.Squire, C. Rogers, R.Hinchliffe and R.Mouton Type of Anesthesia for Endovascular Abdominal Aortic Aneurysm Repair. J Cardiothorac Vasc Anesth.vol.33, pp. 462-471,2019.

[14] F.Menezes, B.Ferrarezi, M.Souza, S.Cosme and G.Molinari Results of Open and Endovascular Abdominal Aortic Aneurysm Repair According to the E-PASS Score. Braz J Cardiovasc Surg.vol.31, pp. 22-30,2016.

[15] H.Krishnamoorthi, H. Jeon-Slaughter, A.Wall, S.Banerjee, B.Ramanan, C.Timaran. Rate of Secondary Intervention After Open Versus Endovascular Abdominal Aortic Aneurysm Repair. J Surg Res.vol.232,pp.99-106, 2018. 
[16] S.Han, Y.Kim, S.Heo, S.Woo, Y.Park, D.Kim : Frequency of concomitant ischemic heart disease and risk factor analysis for an early postoperative myocardial infarction after elective abdominal aortic aneurysm repair. Ann Surg Treat Res.vol.90, pp.171-8,2016.

[17] C.Behrendt, A.Dayama, E.Debus, F.Heidemann, N.Matolo, T.Kölbel . Lower Extremity Ischemia after Abdominal Aortic Aneurysm Repair. Ann Vasc Surg.vol.45,pp. 206-212,2017.

[18] M.Malas, I.Arhuidese, U.Qazi, J.Black, B.Perler and J.Freischlag Perioperative mortality following repair of abdominal aortic aneurysms: application of a randomized clinical trial to real-world practice using a validated nationwide data set. JAMA Surg.vol.149, pp.1217-1338, 2014.

[19] T.Chuter, R.Faruqi, R.Sawhney, L. Reilly, R.Kerlan, C.Canto. Endoleak after endovascular repair of abdominal aortic aneurysm. J Vasc Surg.vol.34, pp.98-105,2001.

[20] S.Mulay, A.Geraedts, M.Koelemay and R.Balm ODYSSEUS study group. Type 2 Endoleak With or Without Intervention and
Survival After Endovascular Aneurysm Repair. Eur J Vasc Endovasc Surg.vol.61,pp.779786,2021

[21] C.Stoneburner, G.Frey and A.Hakaim Endoleak after an Open Abdominal Aortic Aneurysm Repair. Journal of Vascular \& Endo Surgery.vol.45,pp.155-160,2016. .

[22] J.Langenberg, J.Kluytmans, H.de Groot, G.Ho, E.Veen, M.Buimer. Surgical Site and Graft Infections in Endovascular and Open Abdominal Aortic Aneurysm Surgery. Surg Infect (Larchmt).vol.19,pp.120-052,2012.

[23] D.Daye and T. Walker Complications of endovascular aneurysm repair of the thoracic and abdominal aorta: evaluation and management. Cardiovasc Diagn Ther.vol.8, pp.138-156, 2018.

[24] M.Zaki, W.Tawfick, M.Alawy, M.ElKassaby, N.Hynes and S.Sultan Secondary aortoduodenal fistula following endovascular repair of inflammatory abdominal aortic aneurysm due to Streptococcus anginosus infection: A case report and literature review. Int J Surg Case Rep.vol. 5, pp.710-3, 2018. 